\title{
Especialistas de la disciplina del currículo en México: precursores en el estudio y conformación del campo
}

\author{
Specialists in the Discipline of the Currículum in Mexico: Pioneers in the \\ Study and Conformation on the Field
}

\section{Especialistas na disciplina do currículo no México: precursores no estudo e conformação do campo}

\section{Efraín Martínez Ambrosio}

Benemérita Universidad Autónoma de Puebla, México efrain.martinezambrosio@correo.buap.mx https://orcid.org/0000-0003-2441-8658

\section{Resumen}

El presente trabajo tiene el objetivo de reflexionar sobre el papel de los especialistas del campo del currículo en México (desde sus inicios en la década de los 70 hasta la actualidad) a partir de la perspectiva de quienes que se consideran como tales y de quienes han vivido el desarrollo de la disciplina. El método de estudio para este trabajo fue cualitativo, inductivo y exploratorio. La técnica de indagación fue la entrevista en profundidad, la cual se aplicó a cuatro especialistas curriculares para obtener datos sobre el significado de su actividad. El proceso de análisis se dividió en tres fases: reducción de datos cualitativos, análisis descriptivo e interpretación. Las perspectivas señalan que el campo del currículo necesita de nuevos investigadores que se consoliden como autoridades dentro de la disciplina, pues no se puede entrever un relevo generacional. En términos generales, los resultados confirman que pocos investigadores se pueden circunscribir dentro de la categoría de especialista en currículo.

Palabras clave: currículo, disciplina, especialista, México. 


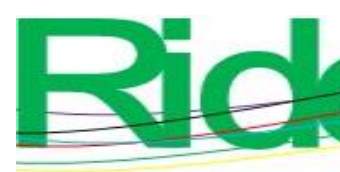

Revista Iberoamericana para la Investigación y el Desarrollo Educativo ISSN $2007-7467$

\begin{abstract}
This paper aims to reflect on the role of specialists in the field of curriculum in Mexico (from its beginnings in the 1970 s to the present) from the perspective of those who consider themselves as such and those who have lived the development of the discipline. The study method for this work was qualitative, inductive, and exploratory. The inquiry technique was the in-depth interview, which was applied to four curricular specialists to obtain data on the meaning of their activity. The analysis process was divided into three phases: qualitative data reduction, descriptive analysis, and interpretation. The perspectives indicate that the field of the curriculum needs new researchers who consolidate themselves as authorities within the discipline, since a generational change cannot be glimpsed. In general terms, the results confirm that few researchers can be circumscribed within the category of curriculum specialist.
\end{abstract}

Keywords: curriculum, disciplines, specialist, Mexico.

\title{
Resumo
}

Este artigo tem como objetivo refletir sobre o papel dos especialistas na área do currículo no México (desde seus primórdios na década de 1970 até os dias atuais) a partir da perspectiva de quem se considera como tal e de quem viveu o desenvolvimento da disciplina. O método de estudo para este trabalho foi qualitativo, indutivo e exploratório. A técnica de investigação foi a entrevista em profundidade, que foi aplicada a quatro especialistas curriculares para obter dados sobre o significado da sua atividade. O processo de análise foi dividido em três fases: redução qualitativa dos dados, análise descritiva e interpretação. As perspectivas indicam que o campo do currículo necessita de novos pesquisadores que se consolidem como autoridades dentro da disciplina, uma vez que não é possível vislumbrar uma mudança geracional. Em termos gerais, os resultados confirmam que poucos pesquisadores podem ser circunscritos à categoria de especialista em currículo.

Palavras-chave: currículo, disciplina, especialista, México.

Fecha Recepción: Febrero 2021

Fecha Aceptación: Agosto 2021 


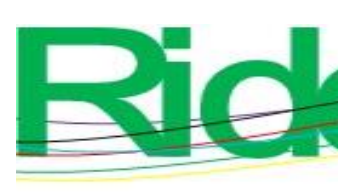

Revista Iberoamericana para la Investigación y el Desarrollo Educativo ISSN 2007-7467

\section{Introducción}

El presente trabajo realiza una reflexión sobre el desarrollo del campo curricular en México, específicamente se centra en realizar una descripción y análisis de las principales tareas y funciones de algunos de los principales especialistas de la disciplina del currículo en el país. Esto nos llevó a identificar momentos históricos que marcaron un hito en el campo y a resaltar la relevancia de estos investigadores en la conformación de la teoría del currículo.

Así, con un enfoque centrado en investigadores reconocidos como autoridades del campo curricular mexicano, considerados especialistas, se plantea una perspectiva diferente en los estudios dominantes en la disciplina.

Es importante aclarar que llamaremos especialistas a las autoridades intelectuales de la disciplina del currículo. Este término ha sido mencionado en algunos trabajos académicos, sin embargo, no ha sido definido en investigaciones $\mathrm{u}$ otras publicaciones. Cabe mencionar que, si insertamos este término en buscadores científicos arbitrados, no aparecen resultados sobre la temática, sino que solo aparecen trabajos que integran los conceptos por separado o concepciones que parecen ser sinónimos, tales como intelectuales o constructor de la palabra.

En México, vinculadas a la educación superior, Glazman y de Ibarrola (1975a, 1975b, 1975c) fueron las primeras en emprender una reflexión sistemática sobre lo que posteriormente se llamaría campo del currículo. De hecho, a partir de ese momento, diferentes investigadores, a través de diversas contribuciones, se fueron consolidando y con el paso del tiempo se les fue reconociendo también como especialistas en esta disciplina.

\section{Aproximaciones conceptuales a la definición de especialista del currículo}

Existen diversas aproximaciones conceptuales al especialista curricular o especialista de la disciplina curricular. Una parte de ellas le adjudica a esta figura un carácter abierto, indeterminado, propositivo y transformador (Martínez, 2017, 2020). Mientras que, con una terminología particular, de Alba (1989) identifica dentro del campo del currículo al constructor de la palabra como aquel que se desarrolla y desplaza a través de conceptualizaciones alternativas, gracias a lo cual contribuye al progreso del campo. Mansilla (2002), por su parte, menciona que habitualmente la categoría de intelectual es utilizada para designar a los productores "independientes" de valores espirituales. Y ahondando en el tema, encuentra dos tipos de intelectuales: por un lado, quienes anhelan una autonomía de pensamiento, por otro, 


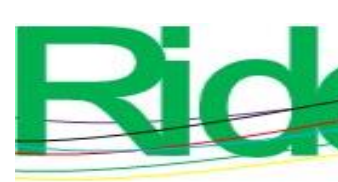

Revista Iberoamericana para la Investigación y el Desarrollo Educativo ISSN $2007-7467$

quienes adoptan ideas, teorías y orientaciones provenientes de países más adelantados. Finalmente, Sánchez y Vega (2003) identifican a quienes realizan un análisis documental, esto es, parten de una búsqueda y recuperación de información primaria para crear información nueva por su forma (secundaria), mediante lo cual solucionan un problema y ordenan el caos. También reconocen a quienes efectúan un análisis informacional, que conlleva un mayor grado de profundidad y valor agregado, donde interviene un especialista de alto nivel en el tema, pues conduce a la creación de algo nuevo, y como resultado soluciona un problema y simplifica lo complejo.

A partir de los anteriores acercamientos conceptuales, puede comprenderse que los especialistas curriculares, especialistas de la disciplina del currículo o especialistas del campo del currículo son intelectuales y constructores de la palabra, pues realizan un análisis documental y posteriormente un análisis informacional. La adopción de ideas, teorías y orientaciones de fuentes primarias representan la base para buscar un pensamiento autónomo y producir algo nuevo. Esto se refleja directamente en la calidad de sus contribuciones al analizar, criticar, construir y realizar nuevas propuestas, las cuales impactan en los contextos local, nacional e internacional.

Como ya lo anticipábamos, los tres tomos de Diseño de planes de estudio de Raquel Glazman y María de Ibarrola (1975a, 1975b, 1975c) conforman la primera propuesta metodológica en la literatura curricular de México. Aquí el plan de estudios se consideraba una de las dimensiones de la planificación universitaria y un instrumento mediante el cual se seleccionaban, organizaban y ordenaban los aspectos de una profesión. Su elaboración requería tomar decisiones sobre los resultados que se pretendían alcanzar con la formación de los estudiantes, la enseñanza de la misma profesión, la duración de los estudios, los recursos a utilizar, el método con que se iba a evaluar el mismo plan de estudios y el aprendizaje de los universitarios.

La metodología propuesta por Glazman y de Ibarrola (1975a) para la elaboración de los planes de estudio hace énfasis en tres aspectos: verificable, sistémica y continua. El método propuesto se puede sintetizar en los siguientes cuatro puntos:

1) La definición del profesional, que se traduce en su mayor capacitación para ejercer una función crítica dentro de la sociedad.

2) La necesidad de definir un modelo de sociedad distinto del modelo de desarrollo aceptado. 


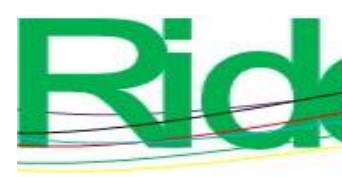

Revista Iberoamericana para la
Investigación y el Desarrollo Educativo
ISSN $2007-7467$

3) La universidad juega un papel relevante en la formación de profesionales con las características deseadas y en la definición del modelo de sociedad buscado.

4) La participación de los estudiantes en el proceso de enseñanza-aprendizaje deberá ser congruente y determinante con la definición profesional y permitir desde el momento mismo de la formación el ejercicio de su responsabilidad social y una función crítica.

Asimismo, Glazman y de Ibarrola (1975a) definen de la siguiente manera al plan de estudios por objetivos de aprendizaje:

Es el conjunto de objetivos de aprendizaje operacionalizados convenientemente, agrupados en unidades funcionales y estructurado de tal manera que normen eficientemente las actividades de enseñanza y de aprendizaje, permitan la evaluación de todo el proceso de enseñanza y aprendizaje, y que conduzca a los estudiantes a alcanzar un nivel universitario de dominio de una profesión (p. 46)

Aunado a ello, Glazman y De Ibarrola (1975a) plantean cuatro apartados dentro de su propuesta:

1) Plan de estudios por objetivos de aprendizaje.

2) Diseño de planes de estudios por objetivos de aprendizaje.

3) El papel de la evaluación.

4) Procesos de la evaluación.

Esta propuesta intentaba ser tan aguda que, en su segundo volumen, describe las operaciones que un equipo de diseño debe efectuar para conformar un plan de estudios: $a$ ) determinar de objetivos generales, $b$ ) determinar de objetivos específicos y $c$ ) agrupación de objetivos específicos y determinación de objetivos intermedios.

Por último, en su tercer volumen, Glazman y de Ibarrola (1975c) destacan cuatro factores a la hora de elaborar planes de estudio. En primer lugar, la estructuración, es decir, el procedimiento para estructurar el plan de estudios, los fundamentos y requisitos. En segundo lugar, la capacitación gradual, la cual apunta a capacitar a los alumnos en actividades específicas, concretas y perfectamente definidas, con el fin de que estos vayan integrando paulatinamente conocimientos profesionales. En tercer lugar, la organización y funciones del cuerpo de diseño, que se enfoca en lograr la integración del trabajo de todo el personal que debe participar en el diseño de un plan de estudios. Finalmente, en el tratamiento de la información se propone un sistema de operación que permite al cuerpo de diseño obtener y 


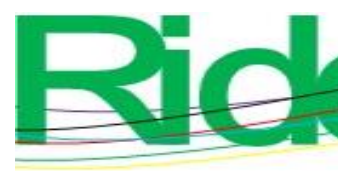

Revista Iberoamericana para la Investigación y el Desarrollo Educativo ISSN $2007-7467$

utilizar de manera rápida la información que se necesite para la elaboración o reforma de un plan de estudios.

En México, a partir de la década de los 70, se profundiza un poco más sobre el campo del currículo y el primer autor que realiza una panorámica sobre el desarrollo de la disciplina a nivel nacional fue Arredondo (1981). Además, de un tiempo para acá, el Consejo Mexicano de Investigación Educativa (Comie) ha realizado estados del conocimiento centrados en el campo curricular, a saber: Procesos curriculares, institucionales y organizacionales (DíazBarriga, 1995), La investigación curricular en México: La década de los noventa (1992-2002) (Díaz-Barriga, 2003) y La investigación curricular en México (2002-2011) (Díaz-Barriga, 2013).

Este trabajo representa la integración de las experiencias, ideas y perspectivas de diferentes especialistas mexicanos que participaron desde un inicio en la investigación curricular de su país. Y aunado a lo anterior, se integran los avances teóricos que se han dado en los últimos años de dicho campo.

\section{Método}

El trabajo adopta un enfoque cualitativo, inductivo y exploratorio, porque se enfoca en comprender y profundizar el fenómeno de estudio, explorando las perspectivas de los participantes en su ambiente natural y en relación con su contexto. Por ello, se decidió elegir la entrevista a profundidad como la técnica de investigación para este estudio, ya que ofrece las condiciones favorables de no distorsionar la información obtenida y conocer con mayor profundidad el papel de los especialistas curriculares.

\section{Objetivos}

El objetivo de este trabajo se centró en comprender las funciones del especialista de la disciplina del currículo, pero no solo a partir de su producción intelectual vertida en los diversos documentos científicos, sino conocer cómo se desenvuelve un experto dentro del campo a través de sus principales tareas, funciones y experiencias. La interrogante que se trazó en esta investigación fue la siguiente: ¿cuál es el papel de los especialistas curriculares en el desarrollo de este campo en México? 


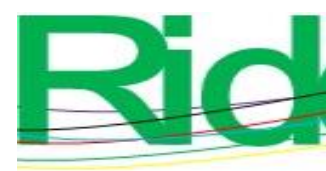

Revista Iberoamericana para la
Investigación y el Desarrollo Educativo ISSN $2007-7467$

\section{Población y muestra}

Hernández, Fernández y Baptista (2014) definen a la muestra de un proceso cualitativo como "el grupo de personas, eventos, sucesos, comunidades, etc., sobre el cual se habrán de recolectar los datos, sin que necesariamente sea estadísticamente representativa del universo o población que se estudia" (p. 384). A los tipos de muestra que usualmente se emplean en las investigaciones de este tipo se les conoce como no probabilísticos o dirigidos, también se les denomina guiados por uno o varios propósitos, pues la elección de los elementos depende de razones relacionadas con las características de la investigación (Hernández et al., 2014). Este muestreo fue propositivo y se invitó a cuatro especialistas mexicanos, quienes respondieron de manera positiva a dicha petición.

Con base en lo anterior, el elemento central son los especialistas de la disciplina del currículo, quienes, con sus perspectivas, facilitan un sentido de comprensión profundo sobre su papel, las problemáticas que enfrentaron desde la génesis del campo y que actualmente confrontan. Los cuatro especialistas del campo curricular son autores mexicanos, cuentan con el grado de doctor, son miembros del Sistema Nacional de Investigadores (SNI), trabajan en instituciones universitarias y se les considera como autoridades intelectuales dentro del área. A continuación, se mencionan a los investigadores que participaron. Cabe especificar que, para identificar sus fragmentos recolectados de las entrevistas, se le asignó un código a cada uno de ellos.

$$
\begin{aligned}
& \text { 1) María Concepción Barrón Tirado } \\
& \text { 2) Ángel Díaz-Barriga Casales } \\
& \text { 3) José María García Garduño } \\
& \text { 4) Édgar Javier González Gaudiano }
\end{aligned}
$$

\section{Instrumento}

El proceso para obtener preguntas obedeció al orden planteado por Hernández et al. (2014): concepto o tópico objetivo, dimensiones y preguntas. El instrumento empleado (ver tabla 1) en esta investigación derivó en diferentes dimensiones (núcleos temáticos), empero, debido a la extensión y relevancia de los resultados, aquí se desarrolla la última dimensión, porque es sustantiva con el objetivo de la investigación y es de los pocos estudios que se centran en estos actores del currículo mexicano. Mentadas dimensiones fueron: 


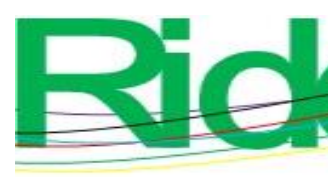

Revista Iberoamericana para la
Investigación y el Desarrollo Educativo
ISSN $2007-7467$

1) Conceptualización del currículo (Martínez, 2020).

2) El desarrollo histórico de la disciplina del currículo en México.

3) Políticas educativas y currículo.

4) Evaluación y currículo.

5) Especialista de la disciplina del currículo (dimensión desarrollada en este trabajo).

Tabla 1. Categoría de análisis de la entrevista en profundidad

\begin{tabular}{|l|l|l|l|}
\hline \multicolumn{3}{|c|}{ El papel de los especialistas del campo curricular en México } \\
(Concepto o tópico objetivo)
\end{tabular}

Fuente: Elaboración propia

Aunado a lo anterior, Martínez (2006) señala que un instrumento empleado en una investigación de este tipo tendrá un alto nivel de validez en la medida en que los resultados reflejen una imagen lo más completa posible y clara de la realidad o situación estudiada, y no solo en aspecto o parte de esta. Y es precisamente la razón por la que esta investigación se apoyó en la entrevista a profundidad, la cual recoge conocimientos, experiencias y aportes de los especialistas que estudian el desarrollo del campo curricular en México, y así refleja la realidad que estos estudiosos han vivido desde el desarrollo de esta disciplina en el país. 


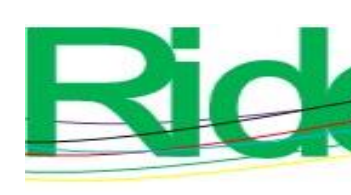

Revista Iberoamericana para la
Investigación y el Desarrollo Educativo ISSN $2007-7467$

\section{Procedimiento de recogida y análisis de datos}

En esta etapa de análisis, la información producida-recolectada que se consiguió a través de las entrevistas en profundidad se transcribió en documentos con formato Word. Posteriormente, se cargaron estos archivos al software ATLAS.ti versión 7.5.7 y se llevó a cabo el proceso de análisis, siguiendo las recomendaciones de Kvale (2011), quien propone tres etapas:

1) La codificación del significado se efectuó asignando una o más palabras clave a un segmento de texto, lo que permitió la identificación de declaraciones (frases) y la categorización de estas por medio de una conceptualización sistémica, esto ayudó a reducir grandes textos.

2) La condensación del significado implica un resumen de los significados expresados por los especialistas curriculares en formulaciones breves. Este análisis plantea cinco pasos:

a) Conseguir una idea general de cada entrevista.

b) Establecer las unidades de significado.

c) Determinar los temas que domina cada unidad de significado.

d) Integrar las unidades de significado desde el punto de vista del propósito específico de esta investigación.

3) En la interpretación del significado se va más allá de lo que se dice directamente para concebir estructuras y relaciones de significado.

A diferencia de la codificación y condensación, que realizan una reducción del texto, las interpretaciones llevan a menudo una expansión del texto. De acuerdo con Kvale (2011): "La interpretación de un texto se caracteriza por un círculo hermenéutico, donde el significado del mismo se establece mediante un proceso en el que se determinan los significados de los distintos pasajes por el significado global del texto" (p. 143). Y teniendo en cuenta lo anterior, se realizó la relectura de los pasajes individuales, pues podían cambiar el significado global del texto anticipado en un primer momento, lo que nuevamente alteraría el significado global de los pasajes individuales, y así repetidamente. La interpretación de las entrevistas fue un proceso circular que concluyó cuando se alcanzó un significado coherente. 


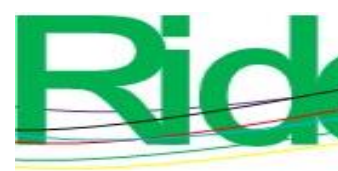

Revista Iberoamericana para la
Investigación y el Desarrollo Educativo ISSN 2007-7467

\section{Resultados}

Las entrevistas permitieron caracterizar la figura del especialista del campo curricular a partir de particularidades que emanan de la experiencia personal y el esfuerzo individual. Los procesos de validación de las inferencias realizadas por los participantes no son evidentes en el estudio, pues no se llevó a cabo, esto significa una limitación del estudio. De manera interesante, uno de los expertos señala la necesidad de nuevos investigadores que se especialicen en esta disciplina, pues considera que el campo se verá desprotegido cuando se retiren estos precursores de la disciplina.

Para el primer entrevistado, los especialistas curriculares en México deben poseer dos saberes fundamentales. En primer lugar, el conceptual y metodológico y, en segundo, el organizativo-proceso de intervención (1:22). Desde esta perspectiva, el experto del campo debe dominar la disciplina tanto teórica como técnicamente.

Yo creo que son dos funciones fundamentales las que se cubren como especialistas en el campo del curricular. Una cuestión conceptualmetodológica, que es el saber específico de la especialidad, de ser especialista en currículum, y la otra, organizativa, más un proceso de intervención para documentar la intervención de los diversos actores en este proceso de evaluación (1:22).

Desde el punto de vista del entrevistado antes citado, el especialista debe dominar su disciplina (1:28) y colaborar con la construcción de nuevas perspectivas teóricas, metodológicas e impulsar el desarrollo del campo (1:29), pero a partir del conocimiento existente (1:32). En sus propias palabras:

Yo creo que el especialista curricular, debe de dominar su disciplina (1:28)

(...) Debe también apoyar a construir otras perspectivas teóricas, metodológicas, de intervención, porque la riqueza para poder seguir avanzando es trabajar en la parte teórica (1:29).

Esta construcción teórica tiene que ser a partir de los saberes que ya se han construido para reorganizarlos, repensarlos y poder buscar alternativas teóricas y metodológicas en el campo (1:32).

La perspectiva anterior hace alusión a dos actividades importantes en los especialistas: teóricos y prácticos que realizan el desarrollo curricular. Al respecto, DíazBarriga (2001) menciona: 

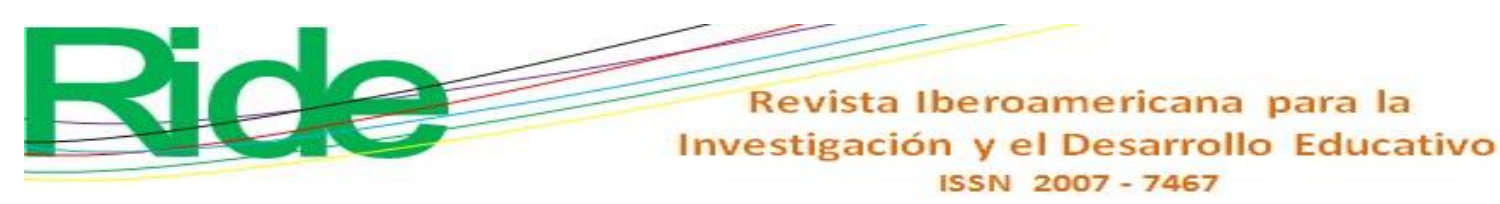

La formación conceptual (o teórica) constituye un baluarte de lo universitario, es la esencia de la misma universidad. Esta formación crea la posibilidad a mediano plazo de un "pensamiento original y creativo", esto es, la producción de conocimientos en un ámbito disciplinario específico. (...) La formación teórica implica la formación para un pensamiento autónomo. Pensamos que dicha formación permite la construcción de conceptos por parte del sujeto, como una acción interna (pp. 43-44).

En esa línea, Alba (1989) sugiere lo siguiente:

El constructor de la palabra (...), el que habla, el que tiene el poder de la palabra en el campo del currículum, es un sujeto social que se mueve y se desarrolla en el contexto de las contradicciones y las luchas propias de este, esto es, de la producción conceptual sobre lo educativo: del campo de la investigación educativa en México (p. 19).

La perspectiva anterior y la cita textual coinciden con la visión del tercer entrevistado, pues también reconoce aspectos fundamentales en el especialista curricular, los cuales son un saber teórico y desarrollo curricular. Además, nombra de forma precisa a ciertos colegas de la disciplina y algunos de sus aportes más importantes. Parte de este momento de la conversación se muestra a continuación:

No sé si por especialistas entiendas los que (...) teorizan sobre el currículum, o los que se dedican al desarrollo curricular. Ángel Díaz-Barriga se ha dedicado a ambos campos; Frida Díaz Barriga se ha dedicado a ambos campos. (...) El último libro de Ángel Díaz-Barriga [Currículum: entre utopía y realidad] es un claro ejemplo en que se conjuga la teoría con el desarrollo curricular. Es el mejor ejemplo que yo veo de un especialista que conjuga ambos campos (3:30).

Si bien la figura de especialista es reconocida dentro del campo por uno de sus saberes citados (desarrollo teórico), es pertinente especificar, como lo hace el primer entrevistado (1:35), que el trabajo de diseño curricular cuenta con cierta complejidad (1:38):

El especialista del campo curricular lo puedes reconocer, pero no domina todo (1:35).

Yo, como especialista en currículum, no podría determinar todas las variables $\mathrm{y}$ todos los elementos (...). Se requiere de un trabajo 


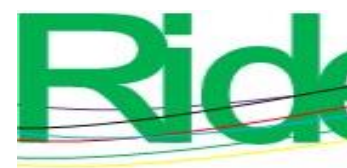

Revista Iberoamericana para la
Investigación y el Desarrollo Educativo
ISSN $2007-7467$

multidisciplinario. El especialista en currículum (...) tiene que recuperar los aportes de las otras disciplinas para acercarse al objeto. (...) Al especialista le toca decir qué se requiere $(1: 38)$.

De manera análoga, Casarini (2013) menciona lo siguiente:

Un modelo de diseño es una representación de ideas, acciones y objetivos, de modo tal que dicha representación sirva como guía a la hora de llevar el proyecto curricular a la práctica. Un modelo de diseño depende del objeto sobre el cual se elabora, así que existen diversos modelos, más o menos prácticos, más o menos teóricos. (...) Los diseñadores experimentan una necesidad de contar con algún andamiaje intelectual para proceder a la tarea del diseño; una especie de constructo previo (...) donde incorporar todos aquellos aspectos considerados pertinentes desde la particular concepción del currículum que se posea; una especie de guía de sus reflexiones para la planeación (pp. 119-120).

Y a modo de complemento, Díaz-Barriga (2015) sostiene:

Los autores que trabajan desde la perspectiva de los planes de estudio son conscientes de la necesidad institucional no sólo de evaluar y reformular los planes, sino también de ofrecer una visión que oriente e invite a los docentes a innovar y organizar su trabajo educativo (p. 25).

Ahora bien, el penúltimo entrevistado se cuestiona a sí mismo: ¿a qué se le denomina especialista? Y responde describiendo uno de los aspectos que debe poseer y cita a otros colegas del campo; también identifica a otro grupo de agentes que intervienen en la disciplina, a quienes denomina técnicos (de lo que se puede deducir que estos personajes no logran equipararse a los primeros) (3:29); aunado a lo anterior, este mismo especialista reconoce a otro grupo de expertos que trabajan bajo consigna de manera práctica para construir los planes de estudio de la Secretaría de Educación Pública (SEP) (3:35):

[Especialista] es (...) el teórico del campo del currículum, como puede ser Alicia de Alba, o tanto un teórico como un experto en desarrollo curricular, como puede ser Ángel Díaz-Barriga y Frida Díaz Barriga, o también los académicos, técnicos que se han dedicado al desarrollo del campo en diferentes instituciones en las que trabajan (3:29). 


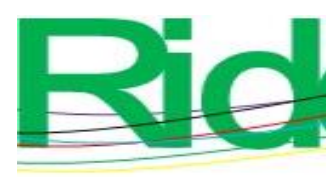

Revista Iberoamericana para la Investigación y el Desarrollo Educativo ISSN $2007-7467$

Y está otro grupo (...), este grupo no ha influido en los planes de estudio de educación básica, lo que hace la SEP es contratar a especialistas directamente del conocimiento y que les ayuden a construir sus nuevos modelos educativos y planes de estudio, pero ese es otro grupo muy grande que se dedica simplemente a la tarea práctica (3:35).

Respecto al segmento (3:35), no está de más confrontarlo con la afirmación de DíazBarriga (2011):

La disociación entre trabajo intelectual y manual es un hecho reconocido sin dificultad. Sin embargo — debido al desarrollo de las fuerzas productivas del conocimiento científico y a las formas de acumulación de capital en la etapa actual del capitalismo - es necesario estudiar la manera como se subdivide del trabajo intelectual, entre quienes realizan la producción de conocimiento, y a quienes les compete solo ser ejecutores técnicos de diversos proyectos profesionales (p. 43).

En esta visión tan compleja, el entrevistado anterior destaca que son pocos los especialistas en el país que cumplen con un papel tan importante (3:31) y propone una definición del especialista:

El grupo de especialistas que tu circunscribes es muy reducido (3:31).

Si definimos especialista, idealmente yo lo vería como aquel que teoriza sobre el campo, sobre su desarrollo y crítica el statu quo del currículum, o sea, el currículum tiene, incluso desde Tyler, una fuerte dosis de crítica. No se puede explicar el desarrollo de la disciplina si no hay una crítica al status quo (3:40).

Mansilla (2002), para comprender cómo los especialistas del campo buscan alternativas a través del estudio, propone una visión analítica y propia:

Una de las ambivalencias más importantes que se pueden detectar entre los intelectuales latinoamericanos: el anhelo de autonomía de pensamiento y creación genuina, por un lado, y la adopción de ideas, teorías y orientaciones provenientes de los países más adelantados del Norte, por otro (p. 430).

El segundo especialista reconoce que ninguno de ellos se formó en Estados Unidos o en el extranjero; más bien constituyeron grupos de trabajo y discusión, tomaron seminarios, 


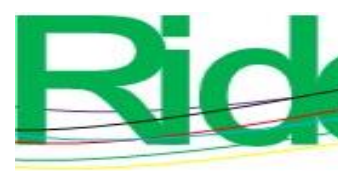

Revista Iberoamericana para la
Investigación y el Desarrollo Educativo
ISSN $2007-7467$

fueron creando sus propios procesos curriculares y cada uno armó escuelas, y cada una de estas ha contado con ámbitos de desarrollo diferentes (2:18). En sus propias palabras:

En general ninguno de nosotros fue a formarse a los Estados Unidos, sino que fuimos haciendo grupos de trabajo, seminarios, grupos de discusión y fuimos armando nuestros procesos curriculares y yo creo que después de eso hemos ido como armando escuelas, y cada escuela ha tenido sus ámbitos de desarrollo (2:18).

Al igual que este, el tercer entrevistado menciona a otros estudiosos de la disciplina que fueron pioneros en la disciplina y a otros más que considera complementarios (3:3). Y específicamente reconoce que un especialista tuvo a una discípula quien propuso el movimiento poscrítico (3:5). Los fragmentos son los siguientes:

Por ejemplo, yo podría reconocer a Alicia de Alba en algún momento, a Frida Díaz Barriga en otro momento, a Conchita Barrón, me podría reconocer yo, podría reconocer a María de Ibarrola y Raquel Glazman, o sea, finalmente hay... Alfredo Furlán me falta y Eduardo Remedi, o sea, hay un grupo de actores curriculares que van teniendo sus desarrollos y van ir teniendo su propio proceso de disolución (2:19)

En México (...) Ángel Díaz-Barriga, Alfredo Furlán, Eduardo Remedi, sobre todo los tres, y complementariamente Patricia Aristi, y otro argentino, Roberto Follari, son los que iniciaron ese campo. A finales de los 70, tuvo una efervescencia en México, se unió el pensamiento curricular argentino con Furlán (3:3).

Una discípula de Díaz-Barriga, que es la Dra. Alicia de Alba, empezó a cuestionar la teoría crítica y eso es lo que se conoce como poscrítico, o es el ingreso del posmodernismo. El posmodernismo cuestiona a la modernización, cuestiona los valores tradicionales de la modernidad (3:5).

En efecto, de Alba (1989) llega a mencionar lo siguiente:

La década de los 70, momento clave en el cual se gesta y empieza a expresarse en México — como sede geopolítica y académica - un movimiento de corte crítico en contraposición a la tendencia tecnologicista dominante en el campo de la educación superior en el país (...), se inicia la construcción del discurso crítico en el campo del currículum. Se critica el diseño curricular por 


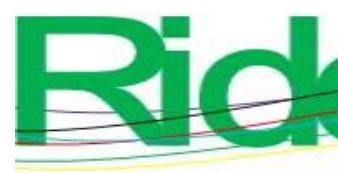

Revista Iberoamericana para la Investigación y el Desarrollo Educativo ISSN $2007-7467$

objetivos, se pone énfasis en los contenidos y en el trabajo de programación a partir de las estructuras conceptuales. En esta tarea se subraya el papel del docente, se intenta la reconceptualización de la noción misma de objetivos y de su vinculación con el problema de acreditación y evaluación (pp. 13-15).

Este mismo entrevistado reconoce que algunos investigadores importantes de la disciplina curricular actualmente ya no volvieron a incursionar en el desarrollo del campo (3:9). Muy preocupado, comenta lo siguiente:

Y algunos han emigrado del campo, como lo ha dicho Alicia de Alba. El mismo Alfredo Furlán, en su tiempo, Eduardo Remedi, Edgar González Gaudiano, emigraron del campo, la misma Raquel Glazman y María de Ibarrola, pues ya se dedican a otras cosas dentro de la educación y no volvieron a incursionar en el desarrollo del campo, está muy limitado (3:9).

En esa misma lógica, el entrevistado anterior menciona que el campo del currículo necesita seguirse desarrollando, pues reconoce una falta de interés por parte de estudiantes, académicos e investigadores (1:39). Similarmente, el primer entrevistado opina que el campo está desprotegido y se requiere de formar a nuevos integrantes (1:40 y 41):

Creo que es un campo que necesita seguirse cultivando, que se requiere seguir trabajando, porque cada vez hay menos estudiantes, académicos, investigadores, interesados en el campo del currículum (1:39).

Necesitamos formar a las nuevas generaciones en este campo (1:40).

El campo del currículum se ha visto desprotegido (1:41).

Esta perspectiva sobre la falta de investigadores especializados en la disciplina curricular en el país se relaciona con el punto de vista del tercer entrevistado, quien opina que, por diferentes razones, existe una crisis en el campo en México. Una de ellas es que no se ha institucionalizado el currículo; otra, que son escasos los grupos investigación, y una más, que no existen estudios de posgrado dedicados de forma específica a esta área de conocimiento (3:28 y 36$)$.

Hay muchas cosas por investigar y los especialistas dedicados al currículum, para un país de este tamaño, son escasos (3:28).

Creo que hay esa crisis en el campo en México (...). Yo lo atribuyo a que no se ha institucionalizado el currículum. Los grupos de investigación son escasos porque no hay, bueno, hay opciones para que los estudiantes hagan 


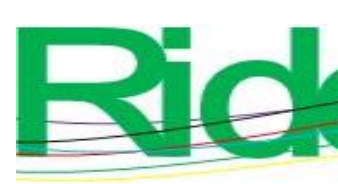

Revista Iberoamericana para la
Investigación y el Desarrollo Educativo
ISSN $2007-7467$

sus tesis sobre currículum, pero no hay una maestría siquiera en currículum, como la tiene Colombia, la tiene Chile, la misma Argentina tiene una en currículum y evaluación, pero nosotros no tenemos ninguna, hay algunos cursos sueltos del currículum en el posgrado, en la licenciatura, pero no, hasta ahí llega (3:36)

Respecto a la perspectiva de una falta de institucionalidad del campo en México, Díaz-Barriga y García (2014) mencionan: "El análisis de la institucionalización del campo puede denotar dos aspectos del campo curricular. El primero, su grado de desarrollo, el segundo, las peculiaridades propias que dicho desarrollo ha tenido en cada país" (p. 262).

Como cierre de esta unidad de análisis, hay un par de reflexiones que vale la pena rescatar:

Lo que tienes que ver aquí son los especialistas de la educación, no solo en el campo del currículum, sino en todo, es tratar de revitalizar estos procesos educativos escolares (4:12).

La revitalización de escuela tiene que darse, pero no a través de modelos educativos como el que nos acaban de presentar hace un par de días; tiene que darse a partir de un debate mucho más serio que emprendamos tanto desde la academia como desde los sindicatos, como desde las distintas esferas, para analizar cuál es el máximo de la escuela... tratarse como una práctica mundial de primer orden político, mundial, científico, tecnológico, etcétera, tenemos que discutir esos puntos y me parece que no se están viendo lo suficiente, el querer acotar las discusiones a los ámbitos o a los espacios que permiten periodos para delimitar es una limitante que tenemos que superar $(4: 14)$.

Los testimonios reconocen la urgencia de una revitalización de la escuela a través de grupos de discusión sobre los modelos educativos planteados y que se analicen por diferentes especialistas, actores, sectores y en espacios que permita superar estos retos mencionados. De acuerdo con Díaz-Barriga (2015):

Es necesario poner en evidencia todas las ilusiones (de la institución educativa) y todas las presiones (de la autoridad) de quienes aspiran a obtener del alumnado solo resultados homogéneos, desconociendo las diferencias socioculturales y personales que existen entre aquellos que asisten a un salón 


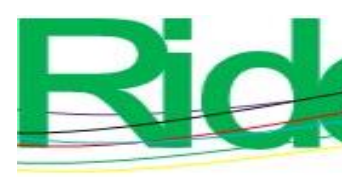

Revista Iberoamericana para la Investigación y el Desarrollo Educativo ISSN $2007-7467$

de clases. (...) En este contexto, en el nuevo siglo se están realizando una serie de reformas curriculares cuyo sentido es necesario analizar (p. 33).

\section{Discusión}

Al de revisar los currículos de los especialistas que participaron en este estudio, se encontró que únicamente José María García Garduño realizó su maestría y doctorado en Estados Unidos; los demás se formaron en nuestro país a través del estudio, de los seminarios e integraron grupos de trabajo y discusión. De esta manera, fueron creando sus propios procesos curriculares y cada uno armó escuelas de pensamiento diferentes. La prueba de todo esto ha sido y son, sin duda, las obras que produjeron y que se consideran referentes para el campo, así como la citación continua de sus nombres en textos académicos, lo que les concede este término específico y privilegiado.

Los especialistas son intelectuales, constructores del discurso y se les reconoce como autoridades en el campo porque han demostrado dominar esta disciplina en dos aspectos fundamentales: teórico y técnico. El primero se refiere a una actividad intelectual que se relaciona directamente con el trabajo teórico-conceptual, es decir, adoptan teorías, metodologías y orientaciones procedentes de otros países, así como el marco referencial de las reformas educativas propias de la nación. Este dominio del campo les permite realizar ensayos teóricos (desarrollos conceptuales), investigaciones de corte conceptual, investigaciones con referentes empíricos y sistematización de experiencias sobre la práctica, que se publican en revistas arbitradas, libros, memorias de congresos, entre otros medios, y de esta forma colaboran en la construcción el discurso curricular. Cabe señalar que inicialmente se formaron a través de la lectura de autores de otros países, pero que paulatinamente fueron desarrollando propuestas asequibles a las necesidades del país.

El especialista en su aspecto técnico es un diseñador curricular. Participa en los procesos de diseño, la mayoría de las ocasiones como asesor, emplea sus conocimientos teórico-metodológicos para organizar y coordinar a los actores que intervienen en un proyecto curricular que se debe diseñar o rediseñar, esto es, la construcción de los fundamentos del nuevo diseño curricular (diagnóstico de necesidades, estudios de egresados, análisis de la práctica profesional, comparación con otros planes de estudio, hasta las etapas de definición del perfil del egresado, de la estructura curricular, dosificación de contenidos y elaboración de programas). 

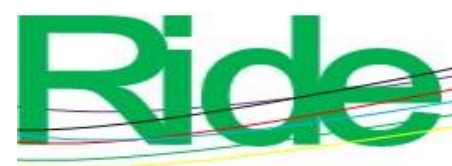

Revista Iberoamericana para la Investigación y el Desarrollo Educativo ISSN 2007 - 7467

\section{Conclusión}

El término especialistas de la disciplina del currículo o especialistas del campo del currículo circunscribe a un número muy reducido de investigadores. Dicha disciplina se enfrenta a una crisis por diferentes razones. Primero, el campo del currículo no se ha institucionalizado, por lo que se requiere la formación de nuevos miembros de la comunidad científica, ya que existen escasos grupos de investigación. Además, no hay en el país estudios de posgrado dedicados a esta área de conocimiento. Por último, se necesita un mayor intercambio internacional con otras comunidades científicas.

Aun con todo, el especialista de la disciplina del currículo domina teórica y técnicamente (diseñador curricular) la disciplina, su papel se reconoce desde un contexto internacional, nacional y hasta local, a través de sus aportaciones conceptuales o teóricas. Además, es reconocido por sus iguales como una autoridad dentro del campo, porque su producción en el campo es un referente de estudio.

\section{Futuras líneas de investigación}

Resulta de manera muy interesante revisar otras temáticas del campo del currículo desde la perspectiva de estos especialistas, por ejemplo, el desarrollo histórico de la disciplina en nuestro país, pues se presentaron a lo largo de los años situaciones políticas, económicas y sociales que fueron configurando el campo del currículo desde la evaluación hasta el diseño curricular. 


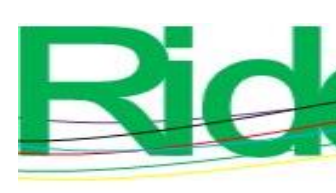

Revista Iberoamericana para la Investigación y el Desarrollo Educativo ISSN $2007-7467$

\section{Referencias}

Arredondo, V. (coord.) (1981). Documento base. Desarrollo curricular. México: Congreso Nacional de Investigación Educativa.

Casarini, M. (2013). Teoría y diseño curricular (3. ${ }^{a}$ ed.). México: Trillas.

de Alba, A. (1989). Del discurso crítico al mito del currículum. En Furlán, A. y Pasillas, M. A. (comps.), Desarrollo de la investigación en el campo del currículum (pp. 13-33). México: Universidad Nacional Autónoma de México.

Díaz-Barriga, A. (coord.) (1995). Procesos curriculares, institucionales y organizacionales. México: Consejo Mexicano de Investigación Educativa.

Díaz-Barriga, A. (2001). Ensayos sobre la problemática curricular (5. ${ }^{a}$ ed.). México: Trillas.

Díaz-Barriga, A. (coord.) (2003b). La investigación curricular en México. La década de los noventa. México: Consejo Mexicano de Investigación Educativa México.

Díaz-Barriga, A. (coord.) (2013). La investigación curricular en México (2002-2011). México: Asociación Nacional de Universidades e Instituciones de Educación Superior-Consejo Mexicano de Investigación Educativa.

Díaz-Barriga, A. (2015). Currículum: entre utopía y realidad. Buenos Aires, Argentina: Amorrortu.

Díaz-Barriga, A. y García, J. M. (coords.) (2014). Desarrollo del currículum en América Latina. Experiencia de diez países. Buenos Aires, Argentina: Miño y Dávila.

Glazman, R. y de Ibarrola, M. (1975a). Diseño de planes de estudio. Vol. I. (2. ${ }^{a}$ ed.). Universidad Nacional Autónoma de México,

Glazman, R. y de Ibarrola, M. (1975b). Diseño de planes de estudio. Vol. II. (2.a ed.). México: Universidad Nacional Autónoma de México.

Glazman, R. y de Ibarrola, M. (1975c). Diseño de planes de estudio. Vol. III. (2. ${ }^{\mathrm{a}}$ ed.). México: Universidad Nacional Autónoma de México.

Hernández, R., Fernández, C. y Baptista, P. (2014). Metodología de la investigación (6. ${ }^{\mathrm{a}}$ ed.). México: McGraw-Hill.

Kvale, S. (2011). Las entrevistas en investigación cualitativa. Madrid, España: Ediciones Morata.

Mansilla, H. C. F. (2002). Intelectuales y política en América Latina. Breve aproximación a una ambivalencia fundamental. Espacio Abierto, 11(3), 429-454. Recuperado de http://www.redalyc.org/articulo.oa?id=12211302. 

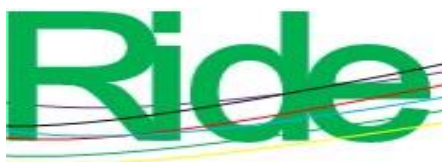

Revista Iberoamericana para la Investigación y el Desarrollo Educativo ISSN $2007-7467$

Martínez, E. (2017). Los especialistas del campo curricular: aproximaciones conceptuales y su elucidación. Revista Dilemas Contemporáneos, 2(17), 1-20. Recuperado de https://www.dilemascontemporaneoseducacionpoliticayvalores.com/index.php/dil emas/article/view/394.

Martínez, E. (2020). La evolución conceptual del término currículo en México: La opinión de tres especialistas. Voces de la Educación, 5(9), 118-128. Recuperado de https://www.revista.vocesdelaeducacion.com.mx/index.php/voces/article/view/19 6.

Martínez, M. (2006). Validez y confiabilidad en la metodología cualitativa. Paradigma, $27(2)$, 7-37. Recuperado

de http://ve.scielo.org/scielo.php?script=sci_arttext\&pid=S101122512006000200002.

Sánchez Díaz, M. y Vega Valdés, J. C (2003). Algunos aspectos teóricos-conceptuales sobre el análisis documental y el análisis de información. Ciencias de la Información, $34(2)$

49-54.

http://132.248.9.34/hevila/Cienciasdelainformacion/2003/vol34/no2/5.pdf 